\title{
Dual-Source Swept-Source Optical Coherence Tomography Reconstructed on Integrated Spectrum
}

\author{
Shoude Chang, Youxin Mao, and Costel Flueraru \\ Institute for Microstructural Sciences, National Research Council Canada, 1200 Montreal Rd, Ottawa, ON, Canada K1A 0R6 \\ Correspondence should be addressed to Shoude Chang, Shoude.chang@nrc.ca
}

Received 26 July 2011; Accepted 9 August 2011

Academic Editor: Nanguang Chen

Copyright () 2012 Shoude Chang et al. This is an open access article distributed under the Creative Commons Attribution License, which permits unrestricted use, distribution, and reproduction in any medium, provided the original work is properly cited.

Dual-source swept-source optical coherence tomography (DS-SSOCT) has two individual sources with different central wavelengths, linewidth, and bandwidths. Because of the difference between the two sources, the individually reconstructed tomograms from each source have different aspect ratio, which makes the comparison and integration difficult. We report a method to merge two sets of DS-SSOCT raw data in a common spectrum, on which both data have the same spectrum density and a correct separation. The reconstructed tomographic image can seamlessly integrate the two bands of OCT data together. The final image has higher axial resolution and richer spectroscopic information than any of the individually reconstructed tomography image.

Optical coherence tomography (OCT) is a powerful imaging technology for producing high-resolution cross-sectional images of the internal microstructure of materials and/or biological samples. It has been widely used in medical imaging and biological testing for more than ten years [1-4]. Swept-source optical coherence tomography (SS-OCT) $[5,6]$ has significant signal-to-noise ratio and speed advantages over time-domain OCT [7-9], in which, the broadband laser swept source plays an important role. The linewidth and output power of the source determinate the imaging depth and sensitivity of an SS-OCT system. The bandwidth of the light source determine the imaging axial resolution. At current stage, most commercial available swept sources have a bandwidth about $100 \mathrm{~nm}$ corresponding to an axial resolution around $7.4 \mu \mathrm{m}$ in air.

In some medical applications, when spectral feature appears at a wavelength differing from the central wavelength of the light source or the photo sensor, it could not be investigated by the single-band OCT system. In order to extract more spectral information and enhance the axial resolution, simultaneously imaging at two distinct spectral regions has been demonstrated by time-domain [10], fullfield [11], and spectral-domain [12, 13] OCT systems. Actually, in all the reported dual-band OCT systems, the two sets of band data are produced from the same light source. In time-domain OCT, as the depth information is obtained by means of depth scanning, both the reconstructed images of different bands have the same image dimensions. An effective and practical method for resolution enhancement in dualbeam time-domain OCT had been reported by Baumgartner et al. [14]. For the spectral-domain OCT, as two bands data produced by the same source, the imaging ranges also have the same depth range, if the spectrum data densities of those two bands (associated with linear CCD array, grating device) are the same.

In SS-OCT, the swept source stimulates the OCT system by a series of wavelengths in a time sequence; a photo detector then collects all the responses as Fourier series components of the testing sample. Because the detector is only sensitive to optical power, it loses the phase information in the reflected/back scattered signal. At any moment, the signal detected by sensor can be written as

$$
\begin{aligned}
I\left(k_{i}\right) \approx & {\left[E_{0}\left(k_{i}\right)+H\left(k_{i}\right) E_{0}\left(k_{i}\right)\right] } \\
& \times\left[E_{0}\left(k_{i}\right)+H\left(k_{i}\right) E_{0}\left(k_{i}\right)\right]^{*}, \quad i=1,2,3,4, \ldots, N,
\end{aligned}
$$

where $E_{0}\left(k_{i}\right)$ is the electrical field with $i_{\text {th }}$ wavenumber sent from the source, $H\left(k_{i}\right)$ represents the sample transfer function, and $I\left(k_{i}\right)$ is the signal generated by the sensor. [ ]* indicates conjugate, and $N$ is the total number of the wavenumbers. Assuming $P_{(k i)}=\left|E_{0}\left(k_{i}\right)\right|^{2}$, power spectrum 


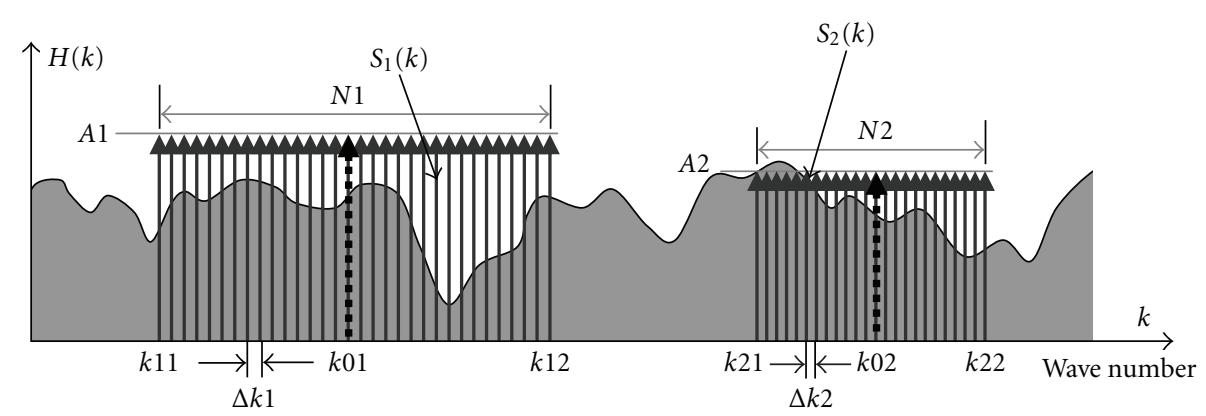

FIgURE 1: Spectrum of two swept sources, $S_{1}(k)$ and $S_{2}(k)$, with different parameters: central wavelength (k01, k02); impulse interval $(\Delta k 1$, $\Delta k 2)$; impulse intensity (A1, A2).

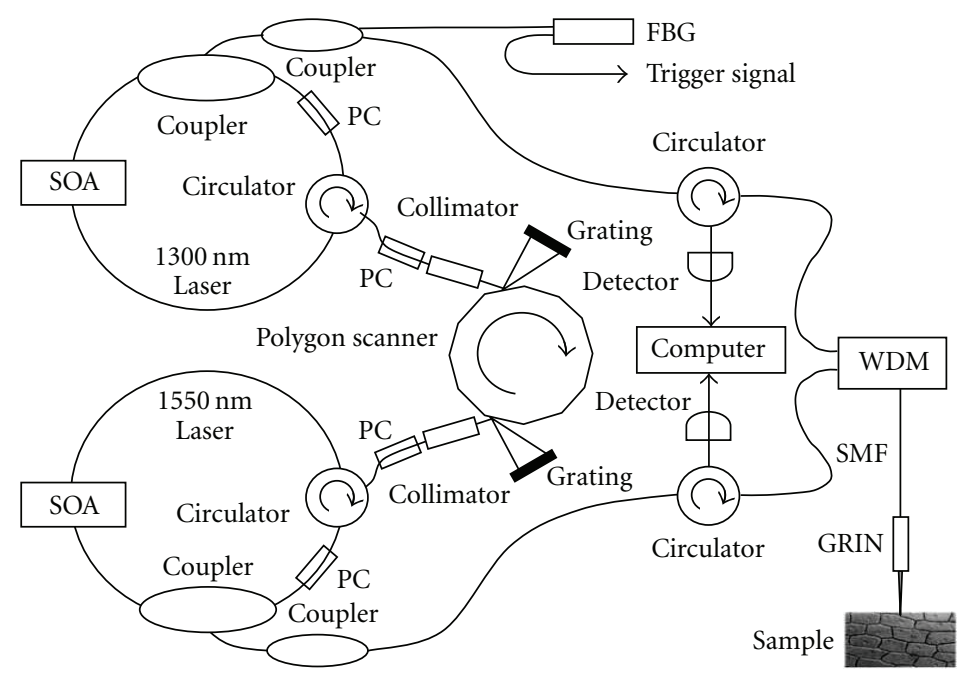

FIGURE 2: Schematic diagram of the dual-source swept-source OCT system. PC: polarization controllers, FBG: fiber Brag grating. SOA: semiconductor optical amplifiers; WDM: wavelength division multiplex; SMF: single-mode optical fiber.

of the light source, the output of OCT system can be expressed by

$$
I_{\left(k_{i}\right)}=P_{\left(k_{i}\right)} H^{2}\left(k_{i}\right)+2 P\left(k_{i}\right) \operatorname{Re}\left[H\left(k_{i}\right)\right]+P\left(k_{i}\right) .
$$

$\operatorname{Re}[H]$ represents the real part of $H$. Assuming $P\left(k_{i}\right)$ is an ideal source which has a constant distribution and $H^{2}\left(k_{i}\right)$ is low frequency component that could be ignored, the processed $I\left(k_{i}\right)$ becomes

$$
I_{p}\left(k_{i}\right) \propto \operatorname{Re}\left[H\left(k_{i}\right)\right] .
$$

The reconstruction of the sample in spatial domain (depth $z$ ) is performed by an inverse Fourier transform:

$$
h(z)=\operatorname{IFT}\left[I_{p}\left(k_{i}\right)\right] .
$$

Because $h(z)$ is the inverse Fourier transform of a real function, it contains a mirror portion. Many works have been done to remove this artifact [15]. The nature of these works is actually to make the intensity signal a complex signal. At any time moment, as (3) and (4) are the results from one wavelength, when all the $I_{p}\left(k_{i}\right)$ s with different wavenumbers form a sequence, the internal structure of the sample can then be extracted by

$$
h(z)=\operatorname{IFT}\left[\Sigma_{n} H\left(k_{i}\right) \delta\left(k_{i}-n\right)\right],
$$

where unit impulse function $\delta\left(k_{i}-n\right)$ is used to separate all Fourier series components with discrete $\mathrm{k}$ numbers.

In SS-OCT, each wavelength independently makes its contribution to the reconstruction as a Fourier series component. This feature gives SS-OCT a possibility to increase the bandwidth by combining multiple swept sources that have different central wavelengths. Figure 1 shows two swept sources, $S_{1}(k)$ and $S_{2}(k)$, with different parameters: central wavelengths or wave numbers $(k 01, k 02)$; spectral-line interval $(\Delta k 1, \Delta k 2)$; impulse intensity (A1, A2). Spectralline interval is given by the sweeping speed of the source and sampling rate of the interface. Impulse intensity is given by the output power of the source.

We have built a dual-source SS-OCT system, which is illustrated in Figure 2 [16]. This OCT system consists of 


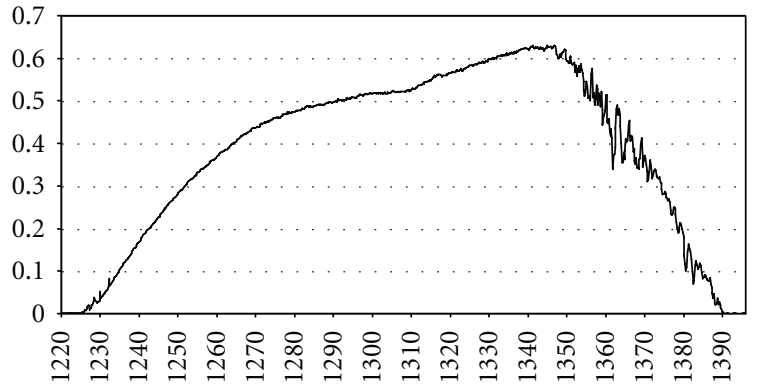

(a) Power spectrum of $1300 \mathrm{~nm}$ source

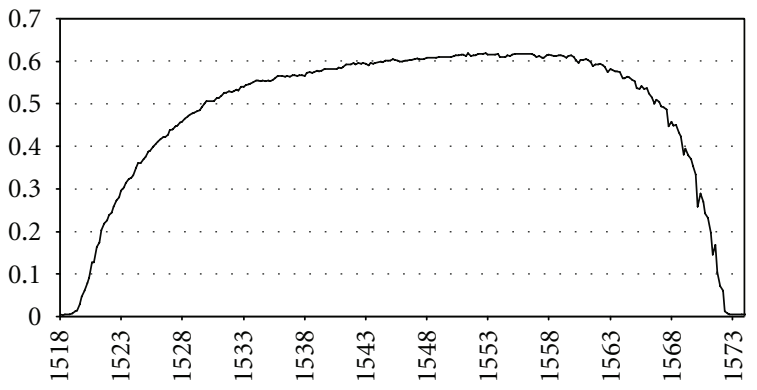

(b) Power spectrum of $1550 \mathrm{~nm}$ source

FIgURE 3: Power spectrums of (a) $1300 \mathrm{~nm}$ swept source; (b) $1550 \mathrm{~nm}$ swept source.

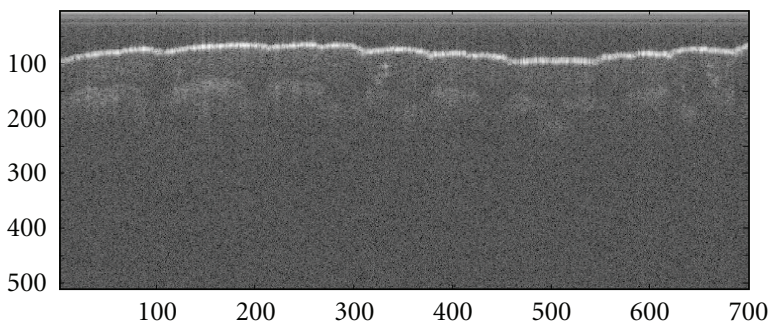

(a) OCT image reconstructed from $1300 \mathrm{~nm}$ source

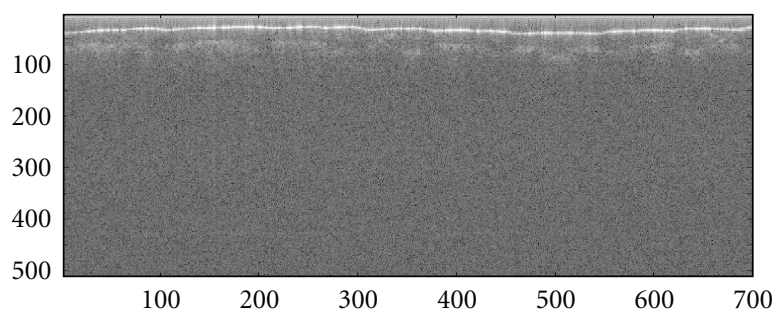

(b) OCT image reconstructed from $1550 \mathrm{~nm}$ source

FIGURE 4: OCT images of a finger. (a) Reconstructed by $1300 \mathrm{~nm}$ swept source; (b) reconstructed by $1550 \mathrm{~nm}$ swept source.

two synchronized swept sources, a wavelength-division multiplexing, and a sharing common path fiber-lensed probe. The dual swept source comprises of two extended ring cavity semiconductor lasers and two high-speed optical narrowband intracavity filters with a single polygonal scanner. The rotating polygon scanner simultaneously tunes the two wavelengths of two lasers synchronously. Two broadband semiconductor optical amplifiers (SOAs) at $1310 \mathrm{~nm} 1550 \mathrm{~nm}$ central wavelengths were used as the cavity gain medium. The inline miniature polarization controller (PC) can reduce cavity lengths. The ratio of the output coupler of 60/40 was used ( $60 \%$ of the power is coupled out) for both cavities, while a $10 \%$ output power of $1310 \mathrm{~nm}$ band was connected to a fiber Brag grating (FBG) the swept trigger signal. The simultaneous swept laser outputs in 1310 and $1550 \mathrm{~nm}$ bands were connected to two matched optical circulators and then connected to a broadband 1310/1550 WDM that outputs two sources into a single-mode optical fiber (SMF). The SMF was fusion-spliced with a home-made GRIN focused lens. The light reflected from the glass-air surface of the GRIN lens, as a reference arm, together with the light reflected from inside sample forms a common-path configuration [17].

The power spectrums of two swept sources are shown in Figures 3(a) and 3(b), and two corresponding OCT images obtained from a human finger are shown in Figures 4(a) and 4(b). It is obvious that two OCT images have different aspect ratios in the imaging depth. In order to merge two OCT images together, one image has to be extended to the same dimension as the other one. One possible way is to find the imaging-depth ratio (IDR) of the two images by comparing two depth ranges of lasers. For a swept source with a Gaussian-profile spectral envelope, the depth range $\Delta z$ is given by [18],

$$
\Delta z=\frac{\lambda_{0}^{2} N_{s}}{(4 n \Delta \lambda)},
$$

where $\lambda_{0}$ is the central wavelength of the swept source, $\Delta \lambda$ is the full-width at half-maximum (FWHM) of the spectral envelope (tuning range), $N_{s}$ is the number of samples within FWHM range of the $\Delta \lambda$, and $n$ is the group refractive index of the sample.

It is difficult and inaccurate to determine the $\Delta \lambda$ by measuring FWHM of the spectrum, when the shape of the spectrum is irregular rather than a Gaussian profile. For example, if the profile slope at the half-maximum position is very low, a small change of the amplitude may introduce a very large change of $\Delta \lambda$ value. As a matter of fact, the IDR of the two sources based on $\Delta \lambda$ s calculated by FWHM does not reflect the real IDR of two reconstructed OCT images due to the inaccurately measured $\Delta \lambda s$. In order to find the real IDR of two sources, we made a simple experiment with the DS-SSOCT system. A small mirror is placed directly in front of the common path probe, and then the number of the interference fringe peaks in OCT signal of each source is counted. The ratio between two numbers represents the IDR of two OCT images.

For the system used in our experiments, the interference peak number from $1300 \mathrm{~nm}$ source is 45 , and peak number from $1550 \mathrm{~nm}$ source is 18 . Therefore, the IDR is 2.5 . To verify this method, we have used a testing sample which has 4-layer glass slice. The A-scan OCT images obtained by 


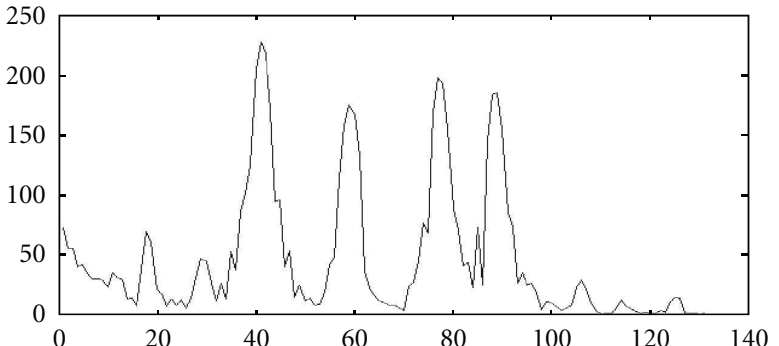

(a) A-scan image obtained by $1550 \mathrm{~nm}$ source

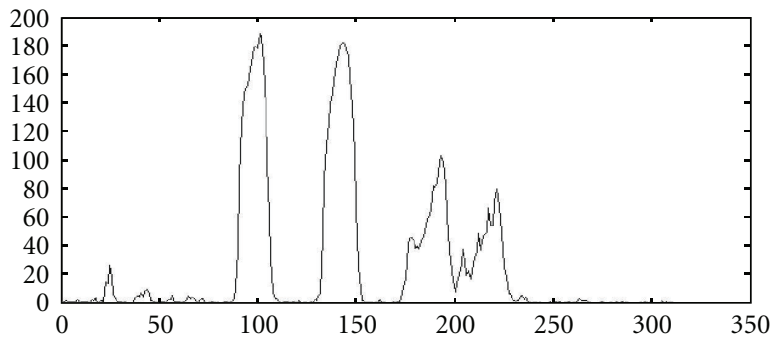

(b) A-scan image obtained by $1300 \mathrm{~nm}$ source

Figure 5: A-scan OCT images of a 4-layer glass sample. (a) A-scan image obtained by $1550 \mathrm{~nm}$ source; (b) A-scan image obtained by $1300 \mathrm{~nm}$ source.

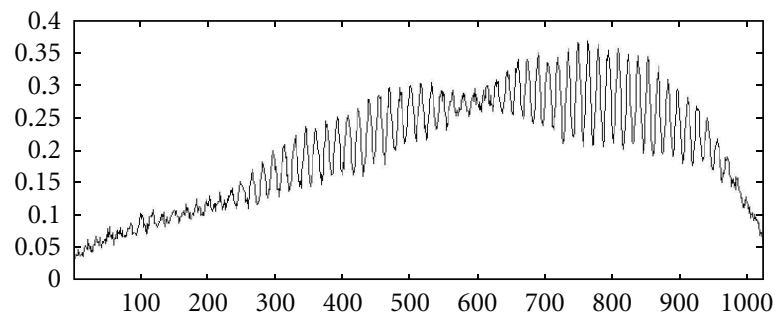

(a) SSOCT signal of finger obtained by $1300 \mathrm{~nm}$ source

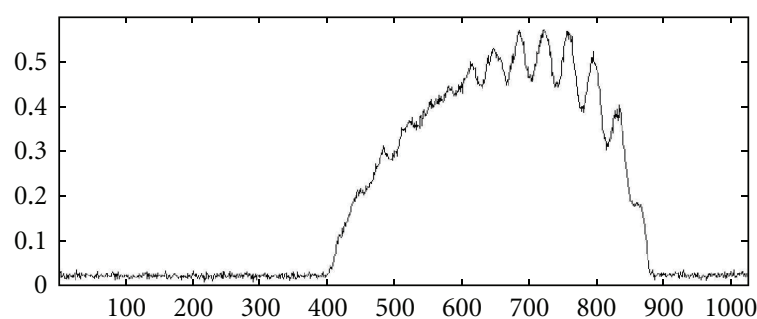

(b) SSOCT signal finger obtained by $1550 \mathrm{~nm}$ source

FIGURE 6: Raw SSOCT signals of finger. (a) SSOCT signal of finger obtained by $1300 \mathrm{~nm}$ source; (b) SSOCT signal finger obtained by $1550 \mathrm{~nm}$ source.

$1550 \mathrm{~nm}$ source and $1300 \mathrm{~nm}$ source are shown in Figures 5(a) and 5(b), respectively. The IDRs of four-peak positions between Figures 5(a) and 5(b) are given by R1 = 101/41 $\cong$ $2.5 ; \mathrm{R} 2=143 / 59 \cong 2.4 ; \mathrm{R} 3=193 / 77 \cong 2.5 ; \mathrm{R} 4=$ $221 / 89 \cong 2.5$. Averagely, we take the IDR as 2.5 .

As mentioned above, SS-OCT has capability to increase the bandwidth by combining multiple swept sources that have different central wavelengths. The integrated dualsource SS-OCT spectrum is given by

$$
I(k c)=a_{1} S_{1}^{\prime}(k c) H(k c)+a_{2} S_{2}^{\prime}(k c) H(k c),
$$

where $k c$ is the new $k$ scale in the integrated discrete spectrum, and $S_{1}^{\prime}(k c), S_{2}^{\prime}(k c)$ are scaled $S_{1}(k), S_{2}(k)$, in the newly integrated spectrum. In order to adjust contribution of each source, the coefficients $\mathrm{a}_{1}, \mathrm{a}_{2}$ are introduced for each source.

According to the spatial-spectral scaling property of Fourier transform, to reduce the size in spatial domain, the size in spectral domain must be increased. For the DS-SSOCT described above, considering the IDR is 2.5, the SSOCT signal obtained by $1300 \mathrm{~nm}$ source should be scaled up by a factor 5, and the SSOCT signal from 1550 $\mathrm{nm}$ should be scaled up by a factor of 2 . Here, to scale up a spectrum by a factor $s$ means to zero-padding $s-1$ zero/s between two neighboring spectral lines. Figures 6 and 7 show the SSOCT signals before and after spectrum scale changes. In Figures 6(a) and 6(b), the spectrums of the two SSOCT signals have different spectral densities: $160 \mathrm{~nm} / 1024$ points and $50 \mathrm{~nm} / 480$ points for 1300 source and 1550 source, respectively. Figures 7 (a) and 7(b) have been scaled up, so they have the same spectrum density, that is, the same wavelength difference $\Delta w$ per interval $\Delta k c$ in the integrated spectrum. Therefore, their reconstructed images have the same depth dimensions. The $\Delta w$ per $\Delta k c$ can be easily calculated by the ending wavelength $w_{e}$ and starting wavelength $w_{s}$ as well as the total spectral lines $n_{s}$ in each source: $\Delta w=\left|w_{e}-w_{s}\right| / n_{s}$. The total spectral separation between two SSOCT signals in the integrated spectrum is given by: $n_{p}=\left|w_{1 e}-w_{2 s}\right| / \Delta w$, where $w_{1 e}$ and $w_{2 s}$ are ending wavelength of SSOCT from source number 1 and starting wavelength of SSOCT from source number 2, respectively.

In the DS-SSOCT described above, for the source number 1, $1300 \mathrm{~nm}$ swept source, $\Delta w=162 \mathrm{~nm} / 1024$ points (see Figure 3); hence, spectral lines per $\mathrm{nm}$ is 6.3 . The wavelength separation between two bands: $1550 \mathrm{~nm}$ $-1300 \mathrm{~nm}=250 \mathrm{~nm}$. Considering the scale-up factor of $1300 \mathrm{~nm}$ source is 5 , the separation spectral lines between two source signals: $250 \times 6.3 \times 5=7875$ points. Figure 8 shows the integrated spectrum of the DS-SSOCT signal $I(k c)$, where the $a 1=a 2=1$, as defined in (7). The final reconstructed OCT image is obtained by

$$
h(z)=\log (|\operatorname{Trunc}\{\operatorname{IFT}[I(k c)]\}|),
$$

where IFT[ ] denotes inverse Fourier transform, Trunc means that, in the reconstructed OCT image, only the central piece of (2) is kept, and all other mirror pieces are truncated off, while $\log ()$ is logarithm processing to nonlinearly emphasize the subtle details.

Figure 9 shows the reconstructed OCT image from the integrated spectrum. Two parts of the SSOCT signals are 


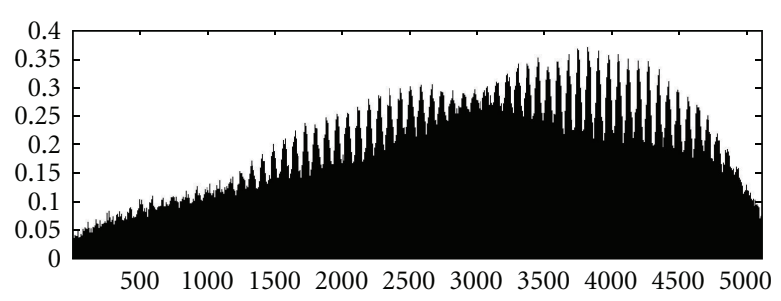

(a) Scaled SSOCT signal obtained by $1300 \mathrm{~nm}$ source

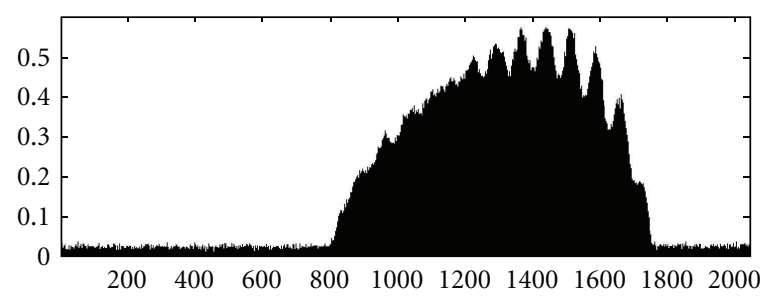

(b) Scaled SSOCT signal obtained by $1550 \mathrm{~nm}$ source

FIGURE 7: Scaled SSOCT signals of Figure 6. (a) scaled SSOCT signal obtained by $1300 \mathrm{~nm}$ source; (b) scaled SSOCT signal obtained by $1550 \mathrm{~nm}$ source.

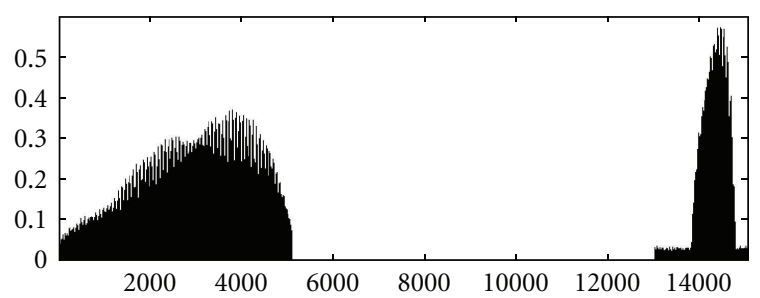

Figure 8: Integrated SSOCT signals obtained from two swept sources.

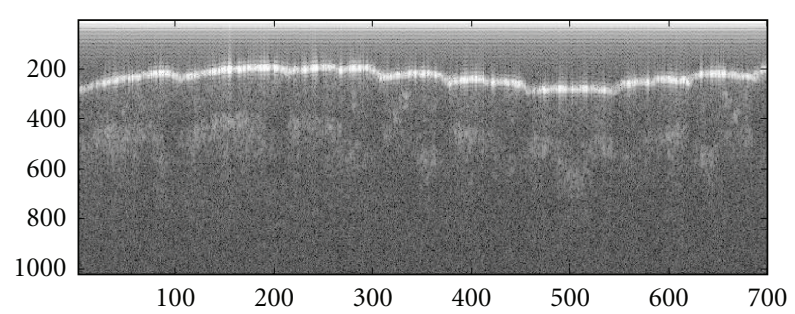

FIGURE 9: OCT image reconstructed from integrated spectrum.

seamlessly integrated together, which has richer spectrum information and depth resolution than any of the individually reconstructed OCT image, since the integrated spectrum has broader bandwidth than other individual two.

In this paper, a new method for integrating the SSOCT signals produced by a dual-source swept-source OCT system is proposed. The resulting OCT image based on the integrated spectrum contains richer spectral and resolution information than any of the individually reconstructed OCT images. This method can be extended to multiple sources of the synchronized swept-source OCT system.

\section{References}

[1] D. Huang, E. A. Swanson, C. P. Lin et al., "Optical coherence tomography," Science, vol. 254, no. 5035, pp. 1178-1181, 1991.

[2] W. Drexler and J. G. Fujimoto, Optical Coherence Tomography, Technology and Applications, Springer, Berlin, Germany, 2008.

[3] G. Smolka, Optical Coherence Tomography:Technology, Markets, and Applications 2008-2012, BioOptics World, Penn Well Corporation, 2008.
[4] U. Morgner, W. Drexler, F. X. Kärtner, X. D. Li et al., "Spectroscopic optical coherence tomography," Optics Letters, vol. 25, no. 2, pp. 111-113, 2000.

[5] S. R. Chinn, E. A. Swanson, and J. G. Fujimoto, "Optical coherence tomography using a frequency-tunable optical source," Optics Letters, vol. 22, no. 5, pp. 340-342, 1997.

[6] S. H. Yun, G. J. Tearney, J. F. De Boer, N. Iftimia, and B. E. Bouma, "High-speed optical frequency-domain imaging," Optics Express, vol. 11, no. 22, pp. 2953-2963, 2003.

[7] M. A. Choma, M. V. Sarunic, C. Yang, and J. A. Izatt, "Sensitivity advantage of swept source and Fourier domain optical coherence tomography," Optics Express, vol. 11, no. 18, pp. 2183-2189, 2003.

[8] J. F. De Boer, B. Cense, B. H. Park, M. C. Pierce, G. J. Tearney, and B. E. Bouma, "Improved signal-to-noise ratio in spectraldomain comared with time-domain optical coherence tomography," Optics Letters, vol. 28, no. 21, pp. 2067-2069, 2003.

[9] R. Leitgeb, C. K. Hitzenberger, and A. F. Fercher, "Performance of Fourier domain vs. time domain optical coherence tomography," Optics Express, vol. 11, no. 8, pp. 889-894, 2003.

[10] F. Spöler, S. Kray, P. Grychtol et al., "Simultaneous dual-band ultra-high resolution optical coherence tomography," Optics Express, vol. 15, no. 17, pp. 10832-10841, 2007.

[11] D. Sacchet, J. Moreau, P. Georges, and A. Dubois, "Simultaneous dual-band ultra-high resolution full-field optical coherence tomography," Optics Express, vol. 16, no. 24, pp. 19434-19446, 2008.

[12] S. Kray, F. Spöler, M. Forst, and H. Kurz, "High-resolution simultaneous dual-band spectral domain optical coherence tomography," Optics Letters, vol. 34, no. 13, pp. 1970-1972, 2009.

[13] P. Cimalla, J. Walther, M. Mehner, M. Cuevas, and E. Koch, "Simultaneous dual-band optical coherence tomography in the spectral domain for high resolution in vivo imaging," Optics Express, vol. 17, no. 22, pp. 19486-19500, 2009.

[14] A. Baumgartner, C. K. Hitzenberger, H. Sattmann, W. Drexler, and A. F. Fercher, "Signal and resolution enhancements in dual beam optical coherence tomography of the human eye," Journal of Biomedical Optics, vol. 3, no. 1, pp. 45-54, 1998.

[15] M. V. Sarunic, M. A. Choma, C. Yang, and J. A. Izatt, "Instantaneous complex conjugate resolved spectral domain and swept-source OCT using $3 \times 3$ fiber couplers," Optics Express, vol. 13, no. 3, pp. 957-967, 2005.

[16] Y. Mao, S. Chang, E. Murdock, and C. Flueraru, "Simultaneous dual-wavelength-band swept source and dual-band common-path optical coherence tomography," Optics Letters, vol. 36, pp. 1990-1992, 2011. 
[17] J. Zhang, B. Rao, L. Yu, and Z. Chen, "High-dynamicrange quantitative phase imaging with spectral domain phase microscopy," Optics Letters, vol. 34, no. 21, pp. 3442-3444, 2009.

[18] F. Lexer, C. K. Hitzenberger, A. F. Fercher, and M. Kulhavy, "Wavelength-tuning interferometry of intraocular distances," Applied Optics, vol. 36, no. 25, pp. 6548-6553, 1997. 

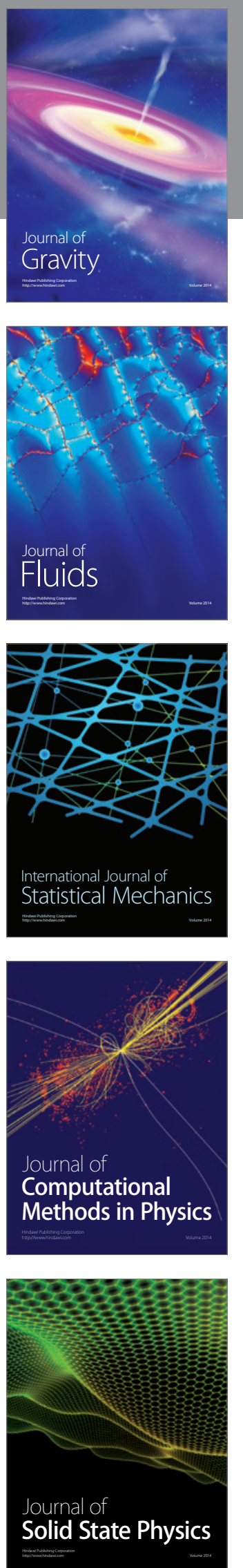

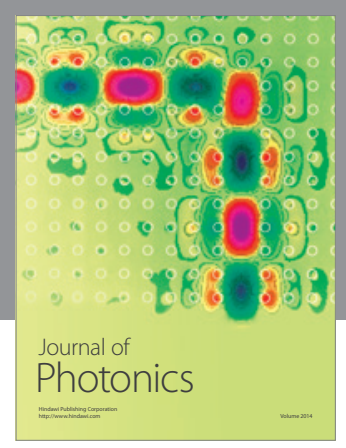

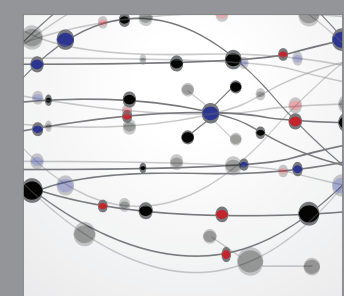

The Scientific World Journal
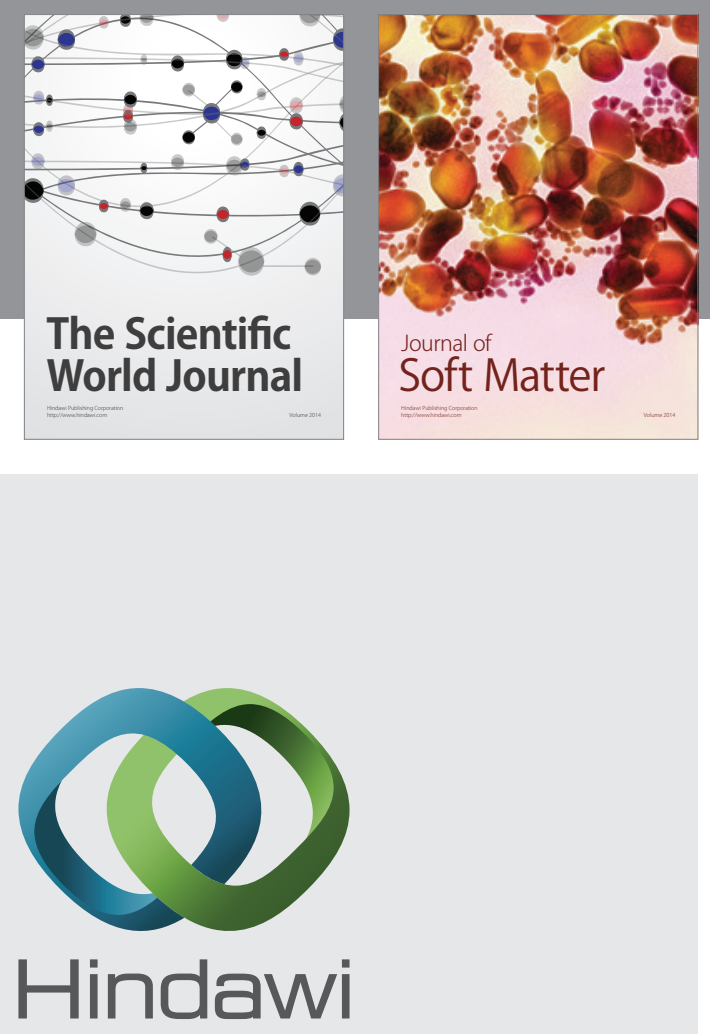

Submit your manuscripts at

http://www.hindawi.com
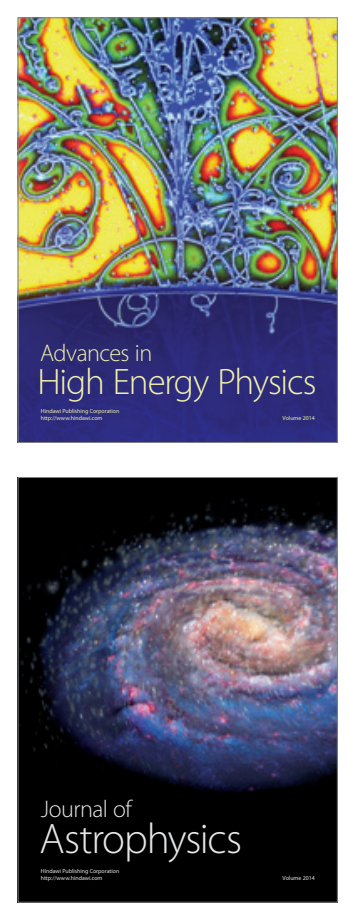
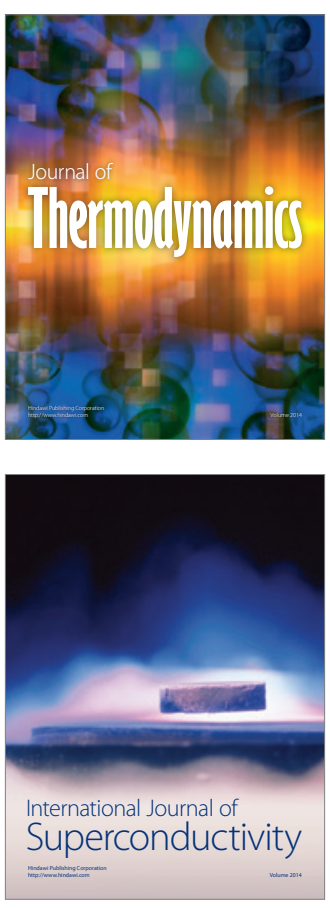
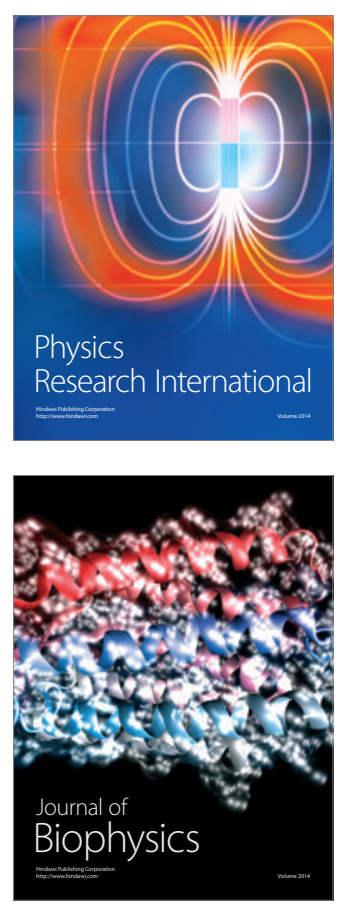
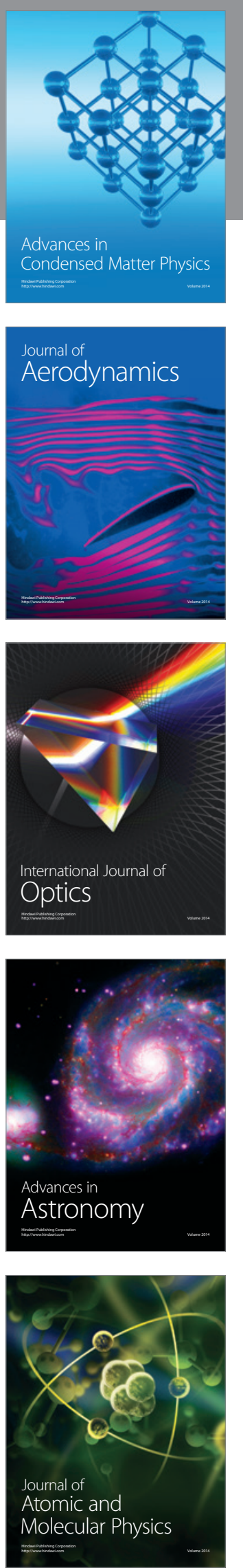